\title{
Assessment of Fetal Lung Maturity by Lamellar Body Count (LBC) in Comparison to the Fluorescence Polarization TDx FLM-II and L/S Ratio Methods
}

Christopher Williams, Pascale Akl and Kenneth E Blick*

Department of Pathology, University of Oklahoma Health Sciences Center, Oklahoma City, USA

\begin{abstract}
Respiratory distress syndrome causes morbidity and mortality of the new-born and is most often associated with premature birth. Laboratory testing can be helpful in establishing fetal lung maturity (FLM) status in situations when premature delivery may be necessary. In addition, the rising incidence of late-preterm births at $>34$ and $<38$ weeks gestation and the associated increases in cost of preterm infant care indicate a need for better preterm delivery FLM assessment. Over the years, the laboratory's primary amniotic fluid FLM screening method included lung surfactant 1) using the lecithin/sphingomyelin ratio (L/S ratio) by thin layer chromatography and 2) the TDx FLM-II method, the latter method being automated and hence available in a timely manner on a $24 / 7$ basis. The lamellar body count (LBC) on amniotic fluid has been shown in studies to be an alternative for FLM assessment however the level of agreement and associated diagnostic cut-offs with LBC with these other classic lung surfactant methods have not been firmly established on our hematology analyzer. We found our LBC method compared favourably with the TDx FLM-II method ( $L B C=0.990^{*} T D x$ FLM-II-3.01; $\left.R^{2}=0.501\right)$ as well as with the classic "gold standard" $L / S$ ratio $\left(L B C=15.2^{*} L / S-3.36\right.$; $\mathrm{R}^{2}=0.762$ ) leading us to conclude that our LBC was an acceptable surrogate for the well-established and no longer available fluorescent polarization TDx FLM-II method for rapid FLM screening. In addition, based on these comparison studies, LBC cut-offs were as follows: Immature, $<=20 \mathrm{k} / \mathrm{mm}^{3}$; Indeterminate, 21 to $49 \mathrm{k} / \mathrm{mm}^{3}$; and mature, $>=50 \mathrm{k} / \mathrm{mm}^{3}$.
\end{abstract}

Keywords: Fetal lung maturity; Respiratory distress syndrome; Lamellar body count; L/S ratio; Late-preterm birth

\section{Abbreviations}

FLM: Fetal Lung Maturity; LBC: Lamellar Body Count; L/S: Lecithin Sphingomyelin Ratio

\section{Introduction}

Respiratory distress syndrome is a significant cause of morbidity and mortality of the new-born, especially those delivered prematurely. The incidence of RDS correlates with immaturity, ranging from approximately 0.3 percent of infants $>38$ weeks gestation to more than 10 percent of late preterm infants defined as $>34$ th weeks of gestation but $<36-6 / 7$ weeks [1]. In the past FLM assessment was extensively utilized in screening deliveries $<38$ weeks gestation, however more recent literature demonstrates that these births are at increased risk of adverse outcome regardless of FLM testing status [2]. Thus, universal testing is no longer recommended. However, as the incidence of latepreterm birth continues to rise along with the high cost of $\mathrm{NO}$ and ECMO therapy, the need for better pre-delivery assessment is essential, especially in the late-preterm group [3,4]. Also, the cost of care for persistence of morbidity in these late-preterm infants throughout the first year of life is three times higher compared to term infants [4]. Therefore, it is important to maintain a laboratory FLM assessment test to support obstetric service in pregnancies that may involve a semielective, medically indicated delivery or uncertain gestational age.

Historically, a number of methods have been used to assess FLM. One of the earliest methods and also considered the "gold standard" is the thin layer chromatographic lecithin/sphingomylein $(\mathrm{L} / \mathrm{S})$ ratio test described in 1971 by Gluck et al. [5]. Several years later, another chromatographic assay for the presence of phosphatidyl-glycerol (PG) was introduced with the advantages that it can be used for vaginal pool samples and in specimens contaminated with maternal blood and meconium [6]. However, these chromatographic methods require a highly skilled technologist and several hours to perform, making the assay rarely available on a timely basis. Several permutations of rapid and easy surfactant screening tests have been introduced over the years including the Shake test, Tap test and Foam Stability test $[7,8]$. While these tests can be useful in identifying infants unlikely to develop RDS, the poor positive predictive value limited the usefulness of these tests as a rapid, 24/7 screening test. Accordingly, the latter surfactant assessment screening tests have fallen out of favor and are rarely performed in most laboratories. Until recently, the most popular and reliable assay was the automated Abbott TDx FLM-II (Abbott Laboratories, Chicago, IL), which measures the surfactant (phospholipid) to albumin ratio [9]. However, the TDx FLM-II was recently discontinued necessitating a need for a replacement method with at least a similar level of accuracy and automation. One option available was the lamellar body count, an assay that measures a stored form of surfactant in the amniotic fluid using a standard hematology analyser [10-14].

While our laboratory used the TDx FLM-II assay for screening, we maintained the legacy L/S ratio test as a reflex test to be used only when the TDx FLM-II gave an indeterminate result. Therefore, 1) since the Abbott TDx FLM-II automated method has since become no longer available and 2) the non-automated L/S ratio was not an acceptable 24/7 FLM screening option, we elected to use the LBC assay as the replacement method for the expiring TDx FLM-II while maintain the $\mathrm{L} / \mathrm{S}$ ratio as a reflex protocol test when this LBC method yielded

*Corresponding author: Kenneth E Blick, Department of Pathology, University of Oklahoma Health Sciences Center, Oklahoma City, USA, Tel: 405271-7632 E-mail: ken-blick@ouhsc.edu

Received April 12, 2017; Accepted May 16, 2017; Published May 22, 2017

Citation: Williams C, AkI P, Blick KE (2017) Assessment of Fetal Lung Maturity by Lamellar Body Count (LBC) in Comparison to the Fluorescence Polarization TDx FLMII and L/S Ratio Methods. J Neonatal Biol 6: 256. doi:10.4172/2167-0897.1000256

Copyright: (c) 2017 Williams C, et al. This is an open-access article distributed under the terms of the Creative Commons Attribution License, which permits unrestricted use, distribution, and reproduction in any medium, provided the original author and source are credited. 
indeterminate results. While many studies have shown that LBC correlates with clinical outcomes, specific cutoffs relative to the TDx FLM-II and the L/S ratio test had not been firmly established. Hence, the present study's focus is to 1) establish a high degree of correlation of the LBC to our previously established FLM methodologies, i.e., the TDx FLM-II and L/S ratio, then 2) establish specific cut-offs for maturity, immaturity, and the indeterminate range, the latter range requiring reflex testing by $\mathrm{L} / \mathrm{S}$ ratio in accordance with our established protocol.

\section{Methods}

For L/S ratio determination, our laboratory uses the Helena Fetal Tek 200 assay (Helena, Beaumont, TX). This involves an extraction step with a methanol-chloroform mixture followed by separation of lecithin and sphingomyelin by thin layer chromatography. The phospholipid bands are visualized using cupric acetate-phosphoric acid reagents with quantization via integration of peak area of the densitometric scan. An $\mathrm{L} / \mathrm{S}$ ratio greater than or equal to 2.0 indicates maturity while a value less than or equal to 1.5 indicates immaturity. A ratio between 1.5 and 2.0 is considered indeterminate.

The FLM-II assay is performed on the Abbott TDx according to the manufacturer's instructions. The TDx utilizes a homogeneous fluorescence polarization method to determine the surfactant to albumin ratio. Our confirmed cut offs are maturity greater than or equal to $55 \mathrm{mg} / \mathrm{g}$; immaturity was confirmed at less than or equal to 40 $\mathrm{mg} / \mathrm{g}$. Indeterminate FLM values, ranging from 40 to $55 \mathrm{mg} / \mathrm{g}$, require testing using the $\mathrm{L} / \mathrm{S}$ ratio to establish maturity or immaturity.

The LBC in this study was performed on the BCI Coulter LH 780 (Beckman Coulter Inc., Brea, CA) haematology analyzer. This method uses electrical impedance for counting particles passing between two electrodes. Normally on the BCI LH 780, formed elements ranging in size from two to twenty fL are counted as platelets. Lamellar bodies in amniotic fluid are of similar size and hence the BCI LH 780 platelet channel can be used for the LBC.

Historical data from 134 amniotic fluid samples, tested for maturity by L/S ratio and the TDx FLM-II as part of a validation of the TDx method, are included for reference. Our current study examines 51 amniotic fluid samples tested by the TDx FLM-II and BCI LH 780 LBC methods to establish concordance of the two methods. Samples found indeterminate by LBC were reflex tested for maturity using the $\mathrm{L} / \mathrm{S}$ ratio.

\section{Results}

Correlation between the L/S ratio and TDx FLM-II showed acceptable linearity as depicted in Figure 1, especially considering that different methodologies and internal references were being utilized. Regression analysis yielded the following: TDx FLM-II $=14.7^{\star} \mathrm{L} /$ $\mathrm{S}+1.89 ; \mathrm{R} 2=0.640$. Figure 1 illustrates the methods correlates more closely around the clinical decision points for maturity, i.e., $\mathrm{L} / \mathrm{S} \geq 2.0$ and TDx FLM-II $\geq 55 \mathrm{mg} / \mathrm{g}$. Indeed, while immature test results by the TDx FLM-II would occasionally be labelled indeterminate or mature by the L/S ratio, every mature result by the TDx FLM-II method was confirmed as mature by the $\mathrm{L} / \mathrm{S}$ ratio. We felt it is critical to avoid falsely mature results in the rapid screening method since such results could potentially lead to adverse clinical outcomes.

Correlation studies comparing LBC to the TDx FLM-II yielded the linear regression equation with a regression line slope equal to unity (LBC $=0.990^{*}$ TDx FLM-II-3.01; R2 $=0.501$ ), as seen in Figure 2. Although the correlation coefficient between methods was not optimal, the methods do agree acceptably in the clinically relevant ranges (TDx
FLM-II, $<40$ and $>55 \mathrm{mg} / \mathrm{g}$ ). Based on our LBC correlation studies with these two reference methods, we set conservative cut off LBC values of less than or equal to $20 \mathrm{k} / \mathrm{mm}^{3}$ for immaturity and greater than or equal to $50 \mathrm{k} / \mathrm{mm}^{3}$ for maturity. Following our legacy protocol, indeterminate values ranging between 20 to $50 \mathrm{k} / \mathrm{mm}^{3}$ were reflexed to the $\mathrm{L} / \mathrm{S}$ ratio test. Table 1 compares the clinical endpoints of the chosen cut offs for LBC with those of the TDx FLM-II. There was diagnostic agreement in the majority of cases (i.e., LBC/TDx: 11/15 immaturity vs. 22/26 maturity) with the TDx method giving better diagnostic lung maturity discrimination. The LBC also had more values falling into the indeterminate diagnostic range (LBC/TDx: 18/9). The overall diagnostic agreement was 66.7 percent between LBC and TDx FLM-II and there was no statistically significant diagnostic difference between these methods. (Chi Square; $\mathrm{p}=0.119$ ).

Sixteen samples found to be indeterminate by LBC were reflexed to the $\mathrm{L} / \mathrm{S}$ ratio test. On these samples where both $\mathrm{LBC}$ and $\mathrm{L} / \mathrm{S}$ were performed, there was acceptable agreement as depicted in Figure 3 and linear regression yielded the following: $\mathrm{LBC}=15.2^{\star} \mathrm{L} / \mathrm{S}-3.36 ; \mathrm{R} 2=0.762$. When comparing clinical endpoints of the methods, there were no instances of discordance between the LBC and L/S methods. Most importantly, there were no instances where a mature result by $\mathrm{LBC}$ was not confirmed by the $\mathrm{L} / \mathrm{S}$ ratio of 2.0 or greater.

Analytical sensitivity of the LBC was experimentally observed to be $1,000 / \mathrm{mm}^{3}$. Regarding interferences, blood and meconium can interfere with the $\mathrm{LBC}^{9}$; these also interfere with the TDx FLM-II and $\mathrm{L} / \mathrm{S}$ ratio.

\section{Conclusion}

We observe acceptable diagnostic concordance between LBC and TDx FLM-II and L/S ratio methods. However, it did appear that the older, and no longer commercially available TDx surfactant based method gave better diagnostic discrimination than LBC between mature and immature with the LBC showing more patient results falling into the indeterminate ranges. Note that for LBC and TDx FLM-II respectively, 21 to $49 \mathrm{k} / \mathrm{mm}^{3}$ and 41 to $54 \mathrm{mg} / \mathrm{g}$ were set as indeterminate ranges based, in part, by $\mathrm{L} / \mathrm{S}$ ratio comparison data. We

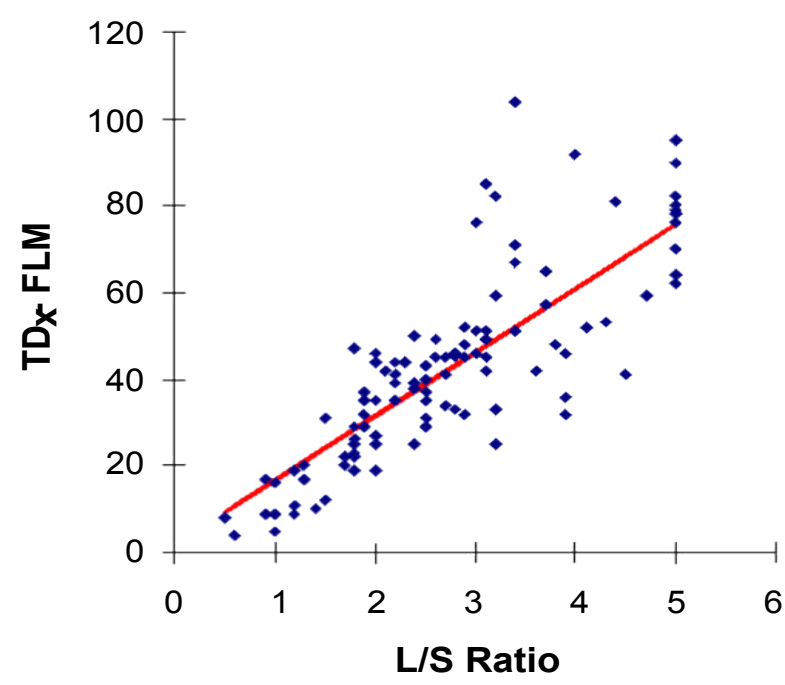

Figure 1: Comparison plot of the TDx FLM-II versus L/S ratio. Linear regression: TDx FLM-II=14.7* L/S + 1.89; $R^{2}=0.640$. 


\section{Lamellar Body Count Versus TDx FLM-II}

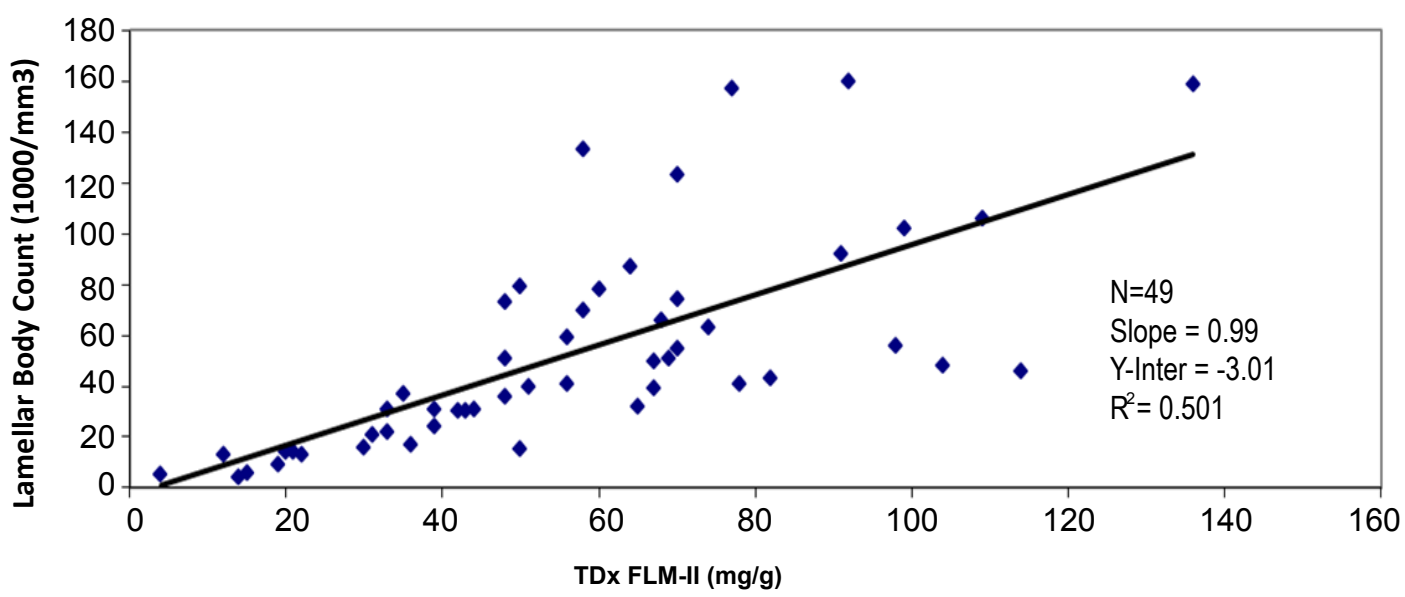

Figure 2: Comparison plot of the Lamellar Body Count versus TDx FLM-II. Linear regression: $L B C=0.99^{*} T D x$ FLM-II $-3.01 ; R^{2}=0.50$.

\begin{tabular}{|c|c|c|c|c|}
\hline & & \multicolumn{3}{|c|}{ TDx FLM-II } \\
\hline & & Immature $<40$ mg/g & Intermediate $41-54 \mathrm{mg} / \mathrm{g}$ & Mature $>55 \mathrm{mg} / \mathrm{g}$ \\
\hline \multirow{3}{*}{ LBC } & Immature $20 \mathrm{k} / \mathrm{mm}^{3}$ & $10(91 \%)$ & $1(9 \%)$ & $0(0 \%)$ \\
\hline & Intermediate $21-49 \mathrm{k} / \mathrm{mm}^{3}$ & $5(28 \%)$ & $6(33 \%)$ & $7(39 \%)$ \\
\hline & Mature $>50 \mathrm{k} / \mathrm{mm}^{3}$ & 0 & $3(14 \%)$ & $19(86 \%)$ \\
\hline
\end{tabular}

Table 1: Concordance of fetal lung maturity assessment based on lamellar body count and the TDx FLM-II.

\section{Lamellar Body Count Versus L/S Ratio}

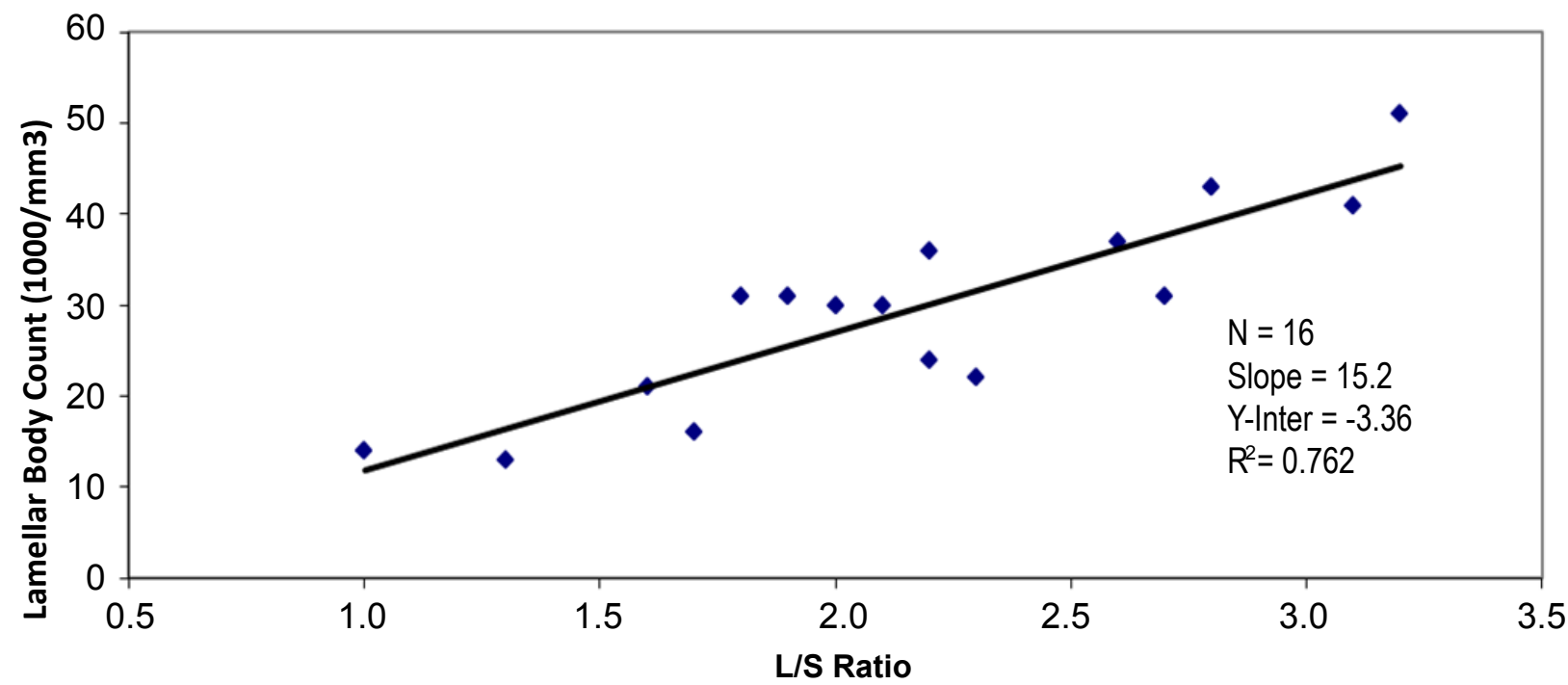

Figure 3: Comparison plot of the Lamellar Body Count versus L/S ratio for samples near the indeterminate range for LBC. Linear regression: $L B C=15.2^{*} \mathrm{~L} / \mathrm{S}-3.36$; $\mathrm{R}^{2}=0.762$. 
Citation: Williams C, Akl P, Blick KE (2017) Assessment of Fetal Lung Maturity by Lamellar Body Count (LBC) in Comparison to the Fluorescence Polarization TDx FLM-II and L/S Ratio Methods. J Neonatal Biol 6: 256. doi:10.4172/2167-0897.1000256

therefore conclude the LBC is clinically acceptable when compared to the older fluorescence polarization TDx FLM-II assay for rapid 24/7 fetal lung maturity assessment. Moreover, we demonstrate that the LBC method shows good diagnostic correlation with the legacy chromatographic $\mathrm{L} / \mathrm{S}$ ratio test.

\section{References}

1. Hibbard JU, Wilkins I, Sun L, Gregory K, Haberman S, et al. (2010) Respiratory morbidity in late preterm births. JAMA 304: 419-425.

2. Kamath BD, Marcotte MP, DeFranco EA (2011) Neonatal morbidity after documented fetal lung maturity in late preterm and early term infants. Am J Obstet Gynecol 204: 518.

3. Loftin RW, Habi, M, Snyder CC, Cormier, CM, Lewis, DF, et al. (2010) Late preterm birth. Rev Obstet Gynecol 3: 10-19.

4. McLaurin KK, Hall CB, Jackson EA, Owens OV, Mahadevia PJ (2009) Persistence of morbidity and cost differences between late-preterm and term infants during the first year of life. Pediatrics 123: 1-12.

5. Gluck L, Kulovich MV, Borer Jr RC, Brenner PH, Anderson GG, et al. (1971) Diagnosis of the respiratory distress syndrome by amniocentesis. Am J Obstet Gynecol 26: 708-710.

6. Hallman M, Kulovich M, Kirkpatrick E, Sugarman RG, Gluck L (1976) Phosphatidylinositol and phosphatidylglycerol in amniotic fluid: Indices of lung maturity. Am J Obstet Gynecol 125: 613-617.
7. Kucuk M (1998) Tap test, shake test and phosphatidylglycerol in the assessment of fetal pulmonary maturity. Int J Gynaecol Obstet 60: 9-14.

8. Thibeault DW, Hobel CJ (1974) The interrelationship of the foam stability test immaturity and intrapartum complications in the respiratory distress syndrome. Am J Obstet Gynecol 118: 56-61.

9. Fantz CR, Powell C, Karon B, Parvin CA, Hankins K, et al. (2002) Assessment of the diagnostic accuracy of the TDx-FLM II to predict fetal lung maturity. Clin Chem 48: 761-765.

10. Caroll S, Towers CV, Wolf L, Duncan L, Weitz B, et al. (2017) The effect of freezing amniotic fluid on lamellar body count fetal lung maturity testing. Int $\mathrm{J}$ Gynaecol Obstet 137: 203-204.

11. Beamon C, Carlson L, Rambally B, Berchuck S, Gearhart M, et al. (2016) Predicting neonatal respiratory morbidity by lamellar body count and gestational age. J Perinat Med 44: 677-83.

12. Tisda H, Kotani T, Sumigama S, Mano Y, Kawabata I, et al. (2015) Amniotic lamellar body count: Predicting and distinguishing neonatal respiratory complications in twin pregnancies. Clin Chim Acta 441: 75-78.

13. Tisda H, Hirakawa A, Kotani T, Sumigama S, Mano $Y$, et al. (2015) Risk assessment for neonatal RDS/TNN using gestational age and the amniotic lamellar body count in twin pregnancies. Clin Chim Acta 451: 301-304.

14. Guven KC, Guvendag Guven ES, Armangil D, Mentese A (2015) Amniotic fluid lamellar body count as a novel biochemical marker for timing elective caesarean delivery J Obstet Gynaecol 35: 451-454. 